\title{
Electrophysiological Evidence for Reversed Lexical Repetition Effects in Language Processing
}

\author{
Tamara Y. Swaab ${ }^{1}$, C. Christine Camblin ${ }^{2}$, and Peter C. Gordon ${ }^{3}$
}

\begin{abstract}
Effects of word repetition are extremely robust, but can these effects be modulated by discourse context? We examined this in an ERP experiment that tested coreferential processing (when two expressions refer to the same person) with repeated names. ERPs were measured to repeated names and pronoun controls in two conditions: (1) In the prominent condition the repeated name or pronoun coreferred with the subject of the preceding sentence and was therefore prominent in the preceding discourse (e.g., "John went to the store after John/he ..."); (2) in the nonprominent condition the repeated name or pronoun coreferred with a name that was embedded in a conjoined noun phrase, and was therefore nonprominent (e.g., "John and Mary went to the store after John/he ..."). Relative to the prominent condition, the
\end{abstract}

nonprominent condition always contained two extra words (e.g., "and Mary"), and the repetition lag was therefore smaller in the prominent condition. Typically, effects of repetition are larger with smaller lags. Nevertheless, the amplitude of the $\mathrm{N} 400$ was reduced to a coreferentially repeated name when the antecedent was nonprominent as compared to when it was prominent. No such difference was observed for the pronoun controls. Because the N400 effect reflects difficulties in lexical integration, this shows that the difficulty of achieving coreference with a name increased with the prominence of the referent. This finding is the reverse of repetition lag effects on $\mathrm{N} 400$ previously found with word lists, and shows that language context can override general memory mechanisms.

\section{INTRODUCTION}

Words have been the subject of much electrophysiological research aimed at understanding language processing in the brain. This emphasis on words parallels that found in behavioral research on language and on human cognition more generally. Psycholinguists often focus on words because all the essential levels of linguistic analysis-phonology, orthography, syntax, and semanticsconnect at the level of words. Cognitive psychologists who study memory often focus on words because they have meaning and vary in familiarity in known ways; also they are very convenient experimentally because they can be presented in different formats (speech or writing) and can be reproduced in those forms. The substantial understanding that has developed from these studies of words provides the basis for addressing a central question about language and the brain, the nature of the relation between higher level language processes and more basic cognitive processes. Even though double dissociations of language and memory function have been found in some amnesias and aphasias, it is not clear whether discourse processing relies on language-specific neural mechanisms or whether it relies on the same neural mechanisms that are used for

${ }^{1}$ University of California-Davis, ${ }^{2}$ Duke University, ${ }^{3}$ University of North Carolina-Chapel Hill

processing episodic and semantic memory. This article uses event-related potential (ERP) methodology to address that question in the domain of reference (where a linguistic expression refers to a semantic entity) and of coreference (where two linguistic expressions refer to the same semantic entity).

Research on words in lists has yielded core experimental phenomena and theoretical constructs in cognitive psychology and cognitive neuroscience. The robust phenomena of frequency effects, repetition priming, and semantic priming have provided the basis of extensive behavioral and electrophysiological investigations (e.g., Kutas \& Hillyard, 1989; Rugg, 1985; Scarborough, Cortese, \& Scarborough, 1977; Meyer \& Schvaneveldt, 1971). Similarly, dissociations between recall and recognition of words constitute core phenomena in memory research (Gillund \& Shiffrin, 1984). Research on words has provided the basis for many commonly accepted mechanisms of cognitive processing. These include such fundamental concepts as the use of familiarity and prior activation for adjusting the thresholds of recognition units (Morton, 1969), the automatic spread of activation to semantically related words (Collins \& Loftus, 1975; Meyer \& Schvaneveldt, 1971), the psychological use of categorical (superset-subset) relations between words (Rips, Shoben, \& Smith, 1973), the use of overlapping meanings to relate words to each other and to facilitate subsequent memory (Bousfield, 1953), and the 
dependence of memory performance on the match between retrieval cues and the memory trace to be retrieved (Gillund \& Shiffrin, 1984). These mechanisms, developed in behavioral research, have been used in interpretations of cognitive processing in the brain (Federmeier \& Kutas, 1999; Osterhout, Bersick, \& McKinnon, 1997; Kounios \& Holcomb, 1992; Rugg, 1990; Rugg \& Doyle 1992; Kutas \& Hillyard, 1989).

Research on words in sentences has shown that lexical frequency, repetition, and semantic relatedness in many instances affect reading or spoken-language comprehension in ways that are analogous to how they affect the recognition of words in lists. This suggests that models of the interpretation of a sentence or passage can in some cases build directly on basic processing mechanisms. For example, the mechanism of spreading activation has been implicated in the way that words with more than one meaning (e.g., "bank") are processed during language comprehension (Seidenberg, Tanenhaus, Leiman, \& Bienkowski, 1982; Swinney, 1979), showing that such mechanisms can help explain the resolution of ambiguity, which is a key requirement of determining the meaning of an utterance. Priming effects have also been found for semantically related word pairs embedded in congruent sentential context (Van Petten, 1993) and repeated words across a congruent discourse context (Van Petten, Kutas, Kluender, Mitchiner, \& McIsaac, 1991).

\section{Coreference in a Discourse Model}

At present it is not agreed upon whether these basic mechanisms of lexical processing can be extended in such a straightforward way to account for the processing of coreference (when two words in the text refer to the same person, e.g., "John went to the store after be/John ..."). At the purely lexical level, coreference can be viewed as a process of matching a word to the memory representation of a previously mentioned word. Viewed this way, it would seem that a repeated word would be the best way to achieve a match because the retrieval cues that it contains exactly fit the representation of the earlier mentioned word, essentially turning coreference into a problem of recognition memory. Indeed, evidence from probe-word recognition tasks (Greene, McKoon, \& Ratcliff, 1992; MacDonald \& MacWhinney, 1990; Gernsbacher, 1989; Chang, 1980; cf. Gordon, Hendrick, \& Foster, 2000) has been used to support this position. The view that a close match between a coreferential expression and its antecedent facilitates the establishment of coreference is described most directly in Gernsbacher's structure building framework (1989, 1990, 1996), which states that coreference with repeated names is achieved more immediately than is coreference with pronouns. Greene et al. (1992) have also argued that repeated-name coreference reflects automatic processing while coreferential pro- cessing of pronouns is not automatic. Both of these perspectives on coreferential processing in language (Greene et al. 1992; Gernsbacher, 1989, 1990, 1996) make explicit connections to concepts developed to account for memory and processing of words that are not presented in sentence context.

In contrast to this work, other evidence from tasks such as reading time (Kennison \& Gordon, 1997; Garrod, Freudenthal, \& Boyle, 1994; Gordon, Grosz, \& Gilliom 1993), acceptability judgments (Gordon \& Hendrick, 1997; Hudson, Tanenhaus, \& Dell, 1986), and priming (Cloitre \& Bever, 1988) does not support the idea that repeating a word is the best way to establish coreference, and, specifically, that establishing coreference with repeated names can be impeded when the referent is prominent in the discourse (e.g., the subject of a sentence). Consideration of the ways in which coreference can be established in a discourse model offers a possible explanation of this difficulty.

The understanding of referential and coreferential expressions is an essential part of the mental construction of a discourse model that embodies the meaning of a sentence or a series of sentences. It is essential both to integrating and differentiating meaning in a discourse model because it is the basis by which the characteristics and actions associated with different expressions that refer to the same entity are integrated together and by which they are simultaneously differentiated from the characteristics and actions associated with different entities mentioned in the text (e.g., Gordon \& Hendrick, 1998; Sanford \& Garrod, 1981; Kintsch \& van Dijk, 1978). The challenges to comprehension posed by coreference are ones that can be analyzed at the level of modeltheoretic semantics.

Model-theoretic semantics analyzes the meaning of a discourse (i.e., a sentence or a group of sentences) in terms of a model of the possible world (or worlds) that the discourse conveys. The critical tasks in building such a model from linguistic input include determining how to represent different types of referential expressions (e.g., indefinite, definite, generic), how to determine which expressions refer to the same discourse referent (i.e., which expressions corefer), and how to understand and represent logical relations (e.g., negation, quantification, entailment). The resulting representation of the meaning of the discourse clearly is not the same as the sum of all the meanings of the individual words in the discourse. Thus, model-theoretic semantics contrasts with lexical semantics, which analyzes the meaning of words in terms of primitive semantic components (e.g., features, attributes) that capture the overlap in meaning of different words.

Recently, model-theoretic semantics has been taken into consideration in psycholinguistic research on reference and coreference (Gordon \& Hendrick, 1998). Of particular relevance to the current article, it has been shown that the prominence of a referent is strongly 
influenced by the syntactic structure of sentences. Specifically, it has been shown that the discourse prominence of a referent can be manipulated by the degree of embedding in a syntactic tree. A referent that is not deeply embedded in a syntactic tree (e.g., the subject of a sentence) is more prominent than one that is more deeply embedded (e.g., when part of a conjoined noun phrase) (Almor, 1999; Gordon et al., 1993; Gordon, Hendrick, Ledoux, \& Yang, 1999). Discourse prominence facilitates coreference with reduced expressions like pronouns but impedes coreference with full expressions like repeated names (Gordon \& Hendrick, 1998).

\section{Neural Processing of Coreference}

Semantic analysis provides conceptual arguments about why the processing of coreference might not build in a straightforward way on general principles of lexical processing but instead might depend on an additional set of processes that serve the function of creating a model of discourse. However, as was discussed before, the behavioral evidence on this question is mixed, with different behavioral paradigms supporting either facilitatory effects of repetition in discourse context, or modulations of the effects of repetition as a function of the discourse context. In the present study, we used ERPs to address this question and to expand understanding of the basic nature of language processing in the brain. ERPs have excellent temporal resolution, and separable ERP components are sensitive to separable aspects of language processing. They therefore can be used to illuminate the component mechanisms of coreferential processing of repeated names in discourse contexts. Of particular relevance to the current research are the N400, the late positive component (LPC), and the $\mathrm{P} 600$ or syntactic positive shift (SPS).

The N400 is a negative-going potential that typically peaks at $400 \mathrm{msec}$ post stimulus and is maximal over posterior electrode sites (Kutas \& Hillyard, 1980). With respect to the processing nature of the $\mathrm{N} 400$, there is evidence that in the context of a discourse, sentence or a word, the modulation of the N400 amplitude is dependent upon the ease with which a word can be integrated into the preceding context (e.g., van Berkum, Hagoort, \& Brown, 1999; Chwilla, Brown, \& Hagoort, 1995; Brown \& Hagoort, 1993; Holcomb, 1993; Van Petten \& Kutas, 1991; Rugg, Furda, \& Lorist, 1988). Its amplitude is reduced to words that are easy to integrate into a higher order representation of the preceding sentence or discourse context (e.g., van Berkum et al., 1999; Van Petten \& Kutas, 1991). In the context of lists of words, a reduced $\mathrm{N} 400$ is found to semantically related and repeated words (e.g., Nagy \& Rugg, 1989; Holcomb, 1993). The N400 repetition effect is modulated by lag, and is smaller when the lag between repeated words increases, both in lists of words and in sentence and discourse contexts (e.g., Van Petten et al., 1991; Nagy \& Rugg, 1989).

Another ERP component sensitive to lexical repetition in word, sentence, and discourse contexts is the LPC. This positive shift peaks around $600 \mathrm{msec}$ and is maximal over posterior electrode sites. Its amplitude is increased to repeated words, but in contrast to the $\mathrm{N} 400$, the LPC is not modulated by increase in lag between repetitions (Nagy \& Rugg, 1989). Amplitude modulation of the LPC has been linked to explicit recall of the first presentation of a repeated stimulus (Paller et al., 1995; Wilding, Doyle, \& Rugg, 1995), such that greater recall of the initial presentation leads to a greater positivity elicited by the repeated word. Of particular importance to the present study is the finding that the LPC is increased to coreferentially repeated names in texts (Van Petten et al., 1991).

The P600/SPS has a latency and scalp distribution similar to that of the LPC, but it can be distinguished from this component because it is sensitive to very different aspects of language processing. Unlike the LPC it is not sensitive to lexical repetition, but instead is modulated by syntactic characteristics of the language input, being prompted by garden-path sentences (Osterhout \& Holcomb, 1992), ungrammatical continuations of sentence fragments (Hagoort, Brown, \& Goothusen, 1993), and dispreferred but grammatical continuations (Kaan \& Swaab, 2003). But of specific importance to the present study is the sensitivity of the P600/SPS to ungrammatical coreference (Osterhout \& Mobley, 1995).

The current experiment uses ERPs to examine if processing the lexical repetition of names that are used as coreferring expressions in a discourse context proceeds independently from that context or, alternatively, if the effects of repetition are modulated by the prominence of a discourse referent. Following Gordon et al. (1999), the current study manipulates discourse prominence by varying the syntactic structure of sentences (see Example 1 below). In the prominent condition, the referent ("John") was a singular noun phrase, whereas in the nonprominent condition the referent was embedded in a conjunctive noun phrase ("John and Mary"). The critical word in the sentence was the second reference, which could either be a repetition of the name "John" or a pronoun "he." The pronoun serves as a semantic control.

(1) John (and Mary) went to the store so that John/he could buy some candy.

Gordon et al. (1999) found that pronouns showed shorter reading times than names for a prominent referent but longer reading times than names for a nonprominent referent.

ERPs to the critical words in these sentences provide a new kind of evidence that can be used to test the competing models of coreference. The idea that general 
principles of lexical processing explain coreference predicts that results for ERPs should follow effects that have been found for memory research using lists of words. The lag effect for word repetition in lists leads to predictions for the nature of $\mathrm{N} 400$ effects for the critical repeated names in this experiment. As discussed previously, the amplitude of the $\mathrm{N} 400$ has been shown to decrease with repeated words, but this attenuation decreases with an increase in lag between the first and second occurrence of the word (e.g., Van Petten et al., 1991; Nagy \& Rugg, 1989), which indicates that integration of repeated words in context is easier with a smaller lag. In the present design this leads to the prediction that the amplitude of the N400 will be reduced in the prominent condition relative to the nonprominent condition because the latter involves a greater lag. In contrast, the discourse-model approach to coreference predicts the exact opposite. According to this approach, integrating a coreferential name (i.e., a repeated name) is easier when the preceding discourse referent is nonprominent, and therefore the amplitude of the $\mathrm{N} 400$ will be reduced to the repeated name when the referent is embedded in a conjunctive noun phrase ("John and Mary") than when it is a singular noun phrase ("John").

With respect to the LPC, no effects of lag are expected. However, Van Petten et al. (1991) have suggested that an increase in the amplitude of the LPC to repeated names in congruent texts can be attributed to increased semantic knowledge about a referent when it is repeated. This leads to the prediction that there will be a greater positivity to the repeated name with a nonprominent referent because embedding the name in the conjunctive noun phrase not only prevents the effects of discourse prominence from occurring, but also increases the number of links in semantic memory that can be made and subsequently retrieved when processing the repeated word.

Finally, within the discourse-model approach, an additional outcome of interest is whether the repeated names in the prominent referent condition show a $\mathrm{P} 600 / \mathrm{SPS}$ as compared to the nonprominent referent condition. This finding would be in the opposite direction of what was predicted for the LPC, and would suggest that repeating a name when the referent is prominent is processed as a grammatical error (Osterhout \& Mobley, 1995).

\section{RESULTS}

Grand average ERPs to the critical names and pronouns in the prominent, singular noun phrase and nonprominent, conjunctive noun phrase conditions are displayed in Figures 1 and 2. ERP data were analyzed using repeated measures analysis of variance (ANOVAs) performed on the mean amplitude of the ERPs to the critical words in the 250- to 500-msec (N400) and 500to 700-msec (LPC and P600/SPS) epochs (relative to a

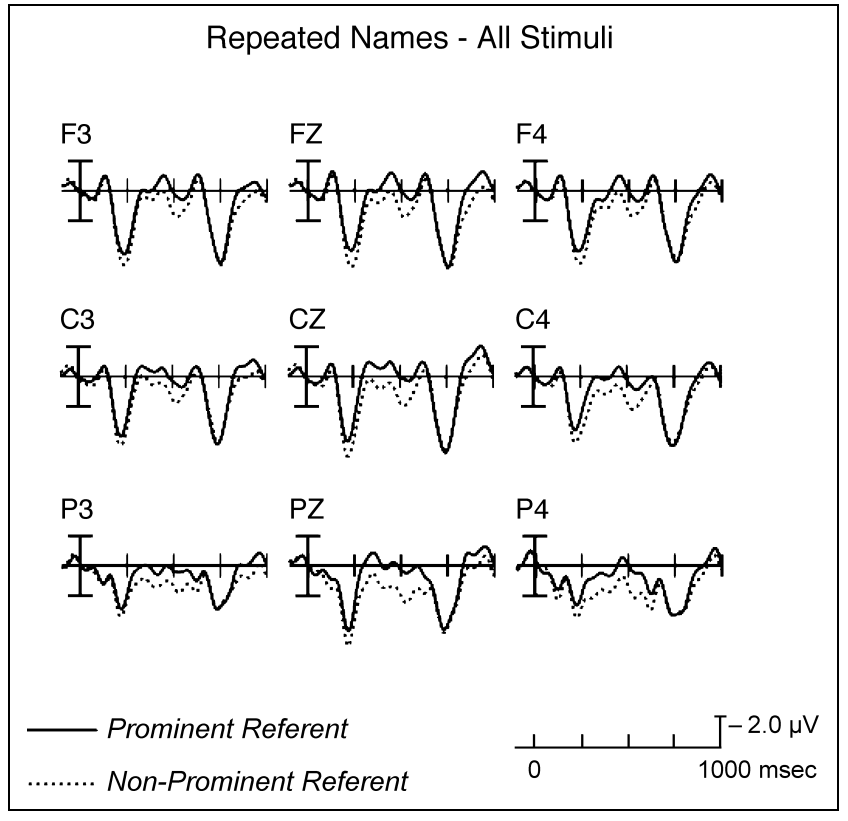

Figure 1. Event-related potentials elicited by repeated names. Repeated names with a prominent referent (i.e., preceded by a single noun phrase) and repeated names with a nonprominent referent (i.e., that were preceded by a conjunction noun phrase) are contrasted. The ERPs are grand averages across all subjects, recorded from frontal (F3, Fz, F4), central (C3, Cz, C4), and posterior sites (P3, Pz, P4).

100-msec prestimulus baseline) at the 30 electrode sites. Separate ANOVAs were done to assess the effects of discourse prominence, the effects of sentential position, and the effects of lexical repetition. For evaluating effects with more than one degree of freedom in the numerator, the Greenhouse-Geisser correction was used to compensate for inhomogeneous variances and covariances across treatment levels (Greenhouse \& Geisser, 1959). The respective adjusted $p$ values are reported.

\section{Discourse Prominence Analysis}

As can be seen in Figure 1, there is a larger negative shift to the repeated names in the prominent condition than in the nonprominent condition in the 250- to 500-msec epoch. This effect has the typical latency and distribution of the N400. The N400 is followed by a positive shift in the 500- to 700-msec time window, which is larger to the repeated names in the nonprominent than in the prominent condition. The latency and topographic distribution of this effect is consistent with both the LPC, which has been observed to lexical repetitions, and the P600/SPS, which has been observed to syntactic manipulations. Figure 2 shows that the ERPs to the pronouns in the prominent and nonprominent conditions mostly overlap. To assess the effects of discourse prominence, two separate ANOVAs with all electrode sites were performed for the 250- to 500- and 500- to 700-msec epochs. Both included the factors of discourse promi- 


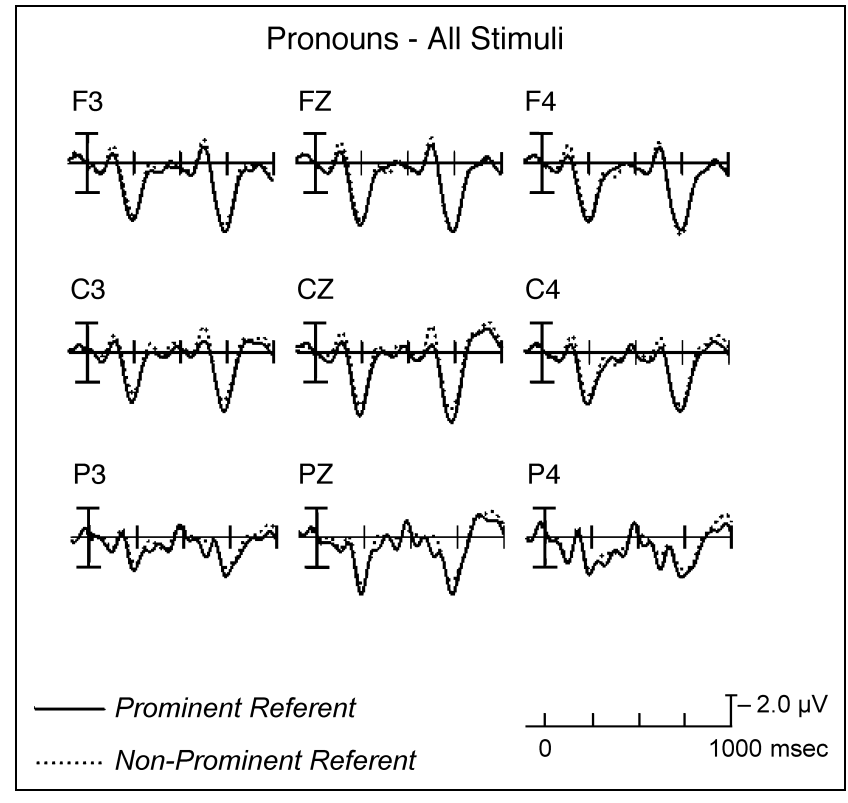

Figure 2. Event-related potentials elicited by pronouns. Pronouns with a prominent referent (i.e., preceded by a single noun phrase) and pronouns with a nonprominent referent (i.e., preceded by a conjunction noun phrase) are contrasted. The ERPs are grand averages across all subjects, recorded from frontal (F3, Fz, F4), central (C3, Cz, C4), and posterior sites (P3, Pz, P4).

nence (prominent, nonprominent), coreferring expression (pronoun, name), and electrode site (30 sites).

\section{0-500 msec}

In this time window there was no main effect of discourse prominence, $F(1,19)=3.65, p=.072$, and no main effect of coreferring expression $(F<1)$, but there was a significant interaction between discourse prominence and coreferring expression, $F(1,19)=5.24, p=.034$. Planned pairwise comparisons were performed separately for repeated name and pronoun comparisons. The amplitude of the $\mathrm{N} 400$ to repeated names was reduced in the nonprominent relative to the prominent condition, $F(1,19)=8.17, p=.010$. This $\mathrm{N} 400$ effect had the typical posterior distribution, as indicated by a significant interaction with electrode site, $F(29,551)=3.64, p=.009$. This pattern of results indicates that despite a shorter repetition lag, it was more difficult to integrate the repeated name in the prominent than in the nonprominent condition. None of the comparisons was significant for the pronouns $(F \mathrm{~s}<1)$. The left side of Figure 3 shows the size of the N400 effect for both names and pronouns.

\section{0-700 msec}

As in the earlier time window, there were no main effects of discourse prominence or coreferring expression, $F<1$ and $F(1,19)=1.74, p=.202$, respectively.
But in contrast to the earlier time window, the interaction between discourse prominence and coreferring expression did not reach significance, $F(1,19)=3.22$, $p=.089$ For the names, the planned pairwise comparisons did not show a significant effect of discourse prominence, $F(1,19)=3.69, p=.070$, but there was a marginally significant interaction between noun phrase and electrode, $F(29,551)=2.28, p=.053$. An analysis of posterior electrodes where the P600/SPS and LPC effects are most prominent showed a significant effect of discourse prominence, $F(1,19)=5.95, p=.025$, such that repeated names with a nonprominent discourse referent elicited more positive ERP traces in this latency window. A planned pairwise comparison of the pronouns did not yield a significant effect of discourse prominence or a discourse prominence by electrode interaction, $F(1,19)=1.06, p=.315$ and $F<1$, respectively.

This direction of the results, that is, a greater positivity for repeated names with a nonprominent referent than for repeated names with a prominent referent, does not fit predictions for the syntactic P600/SPS, but could reflect facilitation of memory retrieval of the referent as a function of more semantic information in the nonprominent conjunctive noun-phrase condition (i.e., LPC). ${ }^{1}$

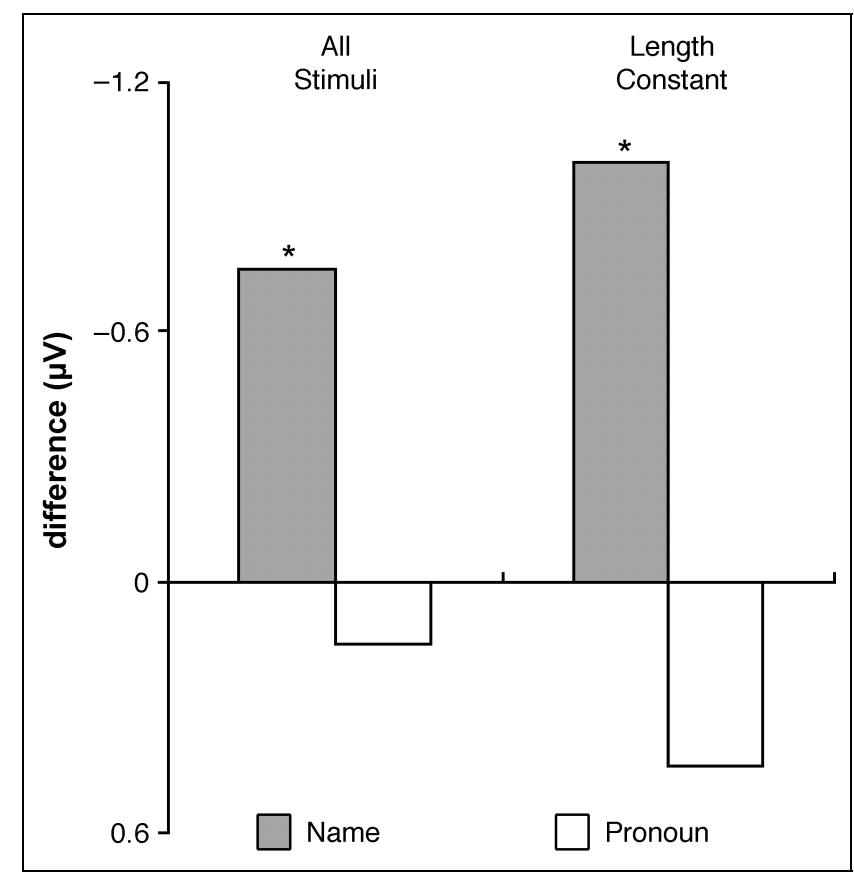

Figure 3. Difference in N400 amplitude. Differences were calculated by subtracting mean voltage amplitude of the ERPs elicited by critical words that were preceded by a prominent referent from the ERPs to critical words that were preceded by a nonprominent referent. On the left is the name versus pronoun comparison for all stimuli. On the right is the name versus pronoun comparison for length constant stimuli. Average amplitude was calculated using all electrodes across all subjects during the 250- to 500-msec latency window. 


\section{Constant Lag Analysis}

The critical words in the nonprominent, conjunctive noun phrase condition systematically occurred two words later in the experimental sentences than the critical words in the prominent, singular noun phrase condition (see Example 1). This possibly confounded effects of discourse prominence with sentence position. To identify possible effects of sentence position, we performed a length constant post hoc analysis, using a subset of the 180 experimental sentences. Two sentence groups were formed: The first group consisted of 56 sentences all of which had 3 to 4 intervening words between the first noun phrase and the critical word (average 3.77), the second group of 86 sentences had 5 to 6 intervening words (average 5.40). By comparing the critical words from the first group in the nonprominent, conjunctive noun phrase condition and critical words from the second group in the prominent, singular noun phrase condition, the main effect of discourse prominence is no longer confounded with sentence position. The critical words were always the seventh or eighth word in the sentence in the prominent and non-prominent conditions, with an average sentence position of 7.40 and 7.77 , respectively. The results of this procedure are displayed in Figures 4 and 5. The size of the N400 effect with the length constant analysis is shown on the right side of Figure 3. As can be seen in these figures, no clear difference in the results between length constant and all stimuli can be observed within the N400 time window.

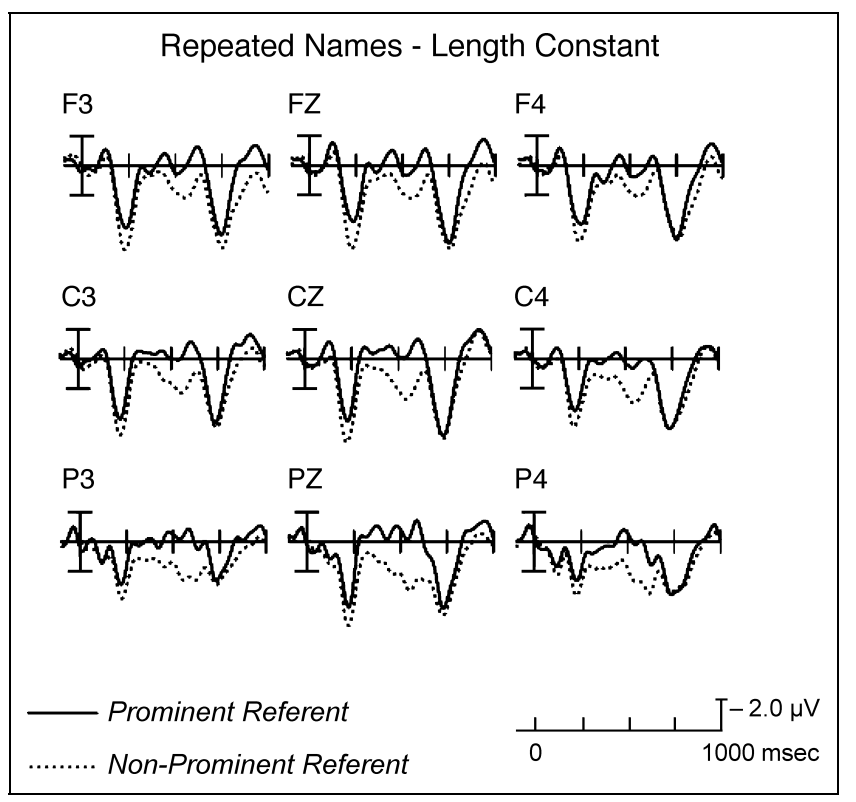

Figure 4. Event-related potentials elicited by repeated names in the length constant analysis. Repeated names with a prominent referent (i.e., preceded by a single noun phrase) and repeated names with a nonprominent referent (i.e., preceded by a conjunction noun phrase) are contrasted. The ERPs are grand averages across all subjects, recorded from frontal $(\mathrm{F} 3, \mathrm{Fz}, \mathrm{F} 4)$, central $(\mathrm{C} 3, \mathrm{Cz}, \mathrm{C} 4)$ and posterior sites (P3, Pz, P4).

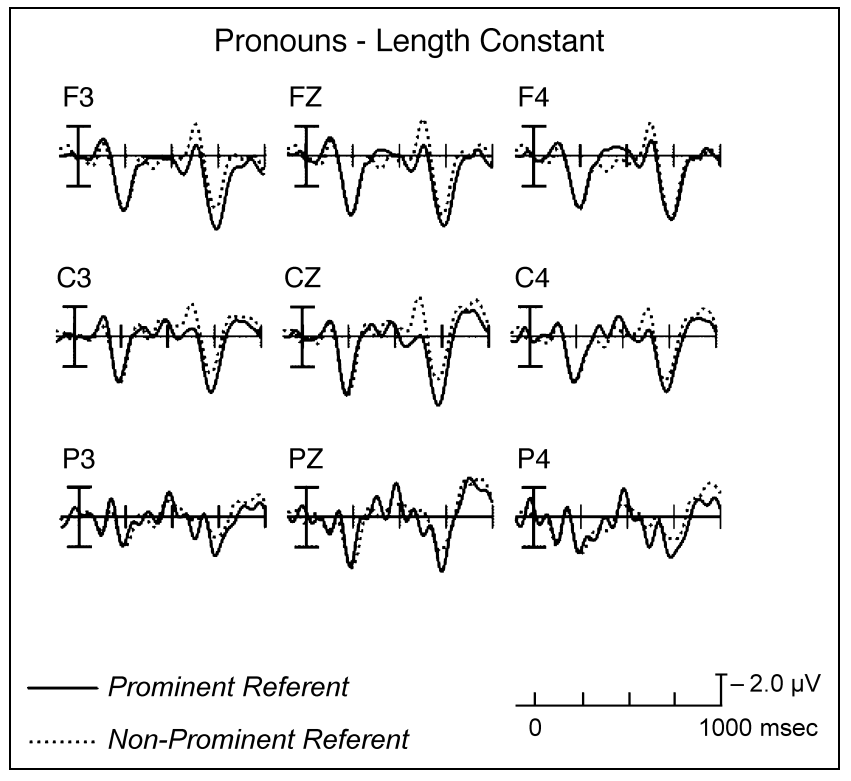

Figure 5. Event-related potentials elicited by pronouns in the length constant analysis. Pronouns with a prominent referent (i.e., preceded by a single noun phrase) and pronouns with a nonprominent referent (i.e., preceded by a conjunction noun phrase) are contrasted. The ERPs are grand averages across all subjects, recorded from frontal (F3, Fz, F4), central (C3, Cz, C4), and posterior sites (P3, Pz, P4).

ANOVAs were compared for the selected length constant sentences in the prominent and the non-prominent conditions in the 250- to 500- and 500- to 700-msec time windows used in the initial analysis.

\section{0-500 msec}

No main effects of discourse prominence or coreferring expression were obtained for the length constant analysis, $F(1,19)=1.04, p=.312$ and $F<1$, respectively, but again a significant interaction between discourse prominence and coreferring expression was found, $F(1,19)=5.57, p=.029$. A planned pairwise comparison for the critical names showed that the ERPs to the names preceded by a prominent referent were significantly more negative than to names preceded by a nonprominent referent, $F(1,19)=4.53, p=.047$. No significant interaction between discourse prominence and electrode site was obtained $(F<1)$, indicating a somewhat more broadly distributed N400 effect than in the ANOVA over all stimuli. As in the initial analysis, none of the comparisons for the critical pronouns were significant: noun phrase: $F(1,19)=1.70, p=.207$; Noun Phrase $\times$ Electrode Site: $F<1$.

\section{0-700 msec}

In this later time window there was also no main effect of discourse prominence or coreferring expression, $F<$ 1 and $F(1,19)=3.59, p=.073$, respectively. Unlike the 
previous analysis of this time window, the interaction between discourse prominence and coreferring expression reached significance, $F(1,19)=7.74, p=.012$. In a planned pairwise comparison of repeated names, a significant effect of discourse prominence was now found, $F(1,19)=5.53, p=.030$, but there was no discourse prominence by electrode interaction, $F(29,551)=1.62, p=.176$. Again, none of the comparisons for pronouns were significant: discourse prominence: $F(1,19)=2.71, p=.116$; discourse prominence by electrode site: $F<1$.

This pattern of results shows that the effects of discourse prominence that we obtained in our initial analyses cannot be attributed to the position of the repeated names in the sentence.

\section{Repeated and Nonrepeated Names Analysis}

Our analysis of the effect of discourse prominence showed a clear pattern that was confirmed in the length constant analysis: a reduction of the $\mathrm{N} 400$ and an increase in the LPC amplitude to the repeated names in the nonprominent relative to the prominent condition. However, in these analyses there were no unrepeated names that could serve as a baseline to directly test the effects of lexical repetition. In order to assess the main effect of lexical repetition, a direct comparison of the repeated names to the first presentation of this name in sentence initial position could not be made, because at this point in the sentence a number of other cognitive processes may have affected the processing of the word that do not play a role when processing words later in the sentence (e.g., arousal and attention). In addition, neuronal refractory effects affect ERPs to words later in the sentence, but not to the sentence initial word. For these reasons, ERPs to words in sentence initial position differ substantially from words later in the sentence (Kutas, 1993), and studies of sentence processing generally do not include ERPs to sentence initial words (e.g., Van Petten and Kutas, 1991). Instead, ERPs to the repeated names were compared with ERPs to the nonrepeated second name in the nonprominent conjoined condition, which was always the third word of the sentence. Because the names that were used in this study were randomly assigned to repetition conditions or to the second name in the conjunctive noun phrase, there is no reason to believe that the names in this comparison differed in a systematic way.

The comparisons discussed here involved three stimulus conditions (unrepeated name, repeated name following prominent referent, and repeated name following nonprominent referent). In order to provide an orthogonal contrast to the comparison between repetition types described above, ERPs to unrepeated names were compared to the average of the ERPs of the repeated names collapsed across discourse prominence.
ERP waveforms for these comparisons are displayed in Figure 6.

\section{0-500 msec}

There was a main effect of repetition, $F(1,19)=42.6, p<$ .001 , a repetition by electrode interaction, $F(29,551)=$ $5.74, p<.001$, and an effect of repetition at posterior electrodes, $F(1,19)=54.23, p<.001$.

\section{0-700 msec}

A main effect of repetition was obtained over all electrodes, $F(1,19)=30.22, p<.0002$, and a significant interaction of repetition by electrode, $F(29,551)=$ $3.65, p<.003$. The effect was also significant over posterior electrode sites, $F(1,19)=20.75, p<.0003 .^{2}$

\section{Summary of Results}

Taken together, the results of the analyses reported above show that the influence of lexical repetition on word processing in the present study was clearly modulated by discourse prominence, such that the reduction of the amplitude of the N400 to the repeated names in the prominent condition was less than to the repeated names in the nonprominent condition, even though the lag between repetitions was smaller in the prominent condition. Conversely, the LPC effect was larger in the

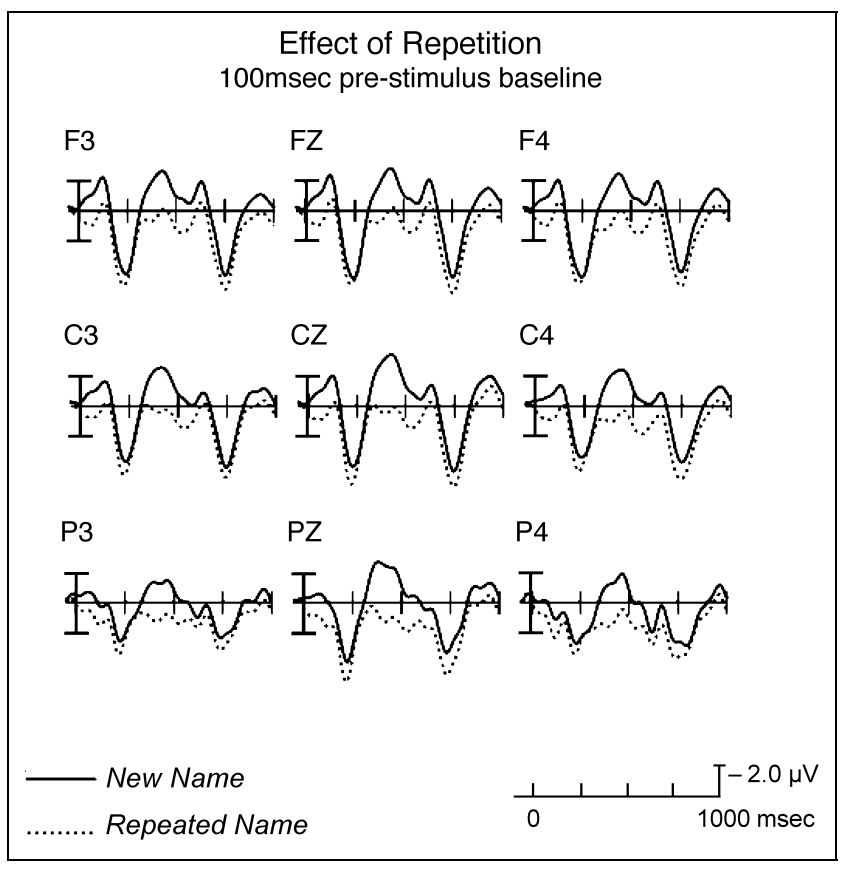

Figure 6. Event-related potentials elicited by new names and repeated names. Repeated names (collapsed across discourse prominence) and new names are contrasted. The ERPs are grand averages across all subjects, recorded from frontal (F3, Fz, F4), central (C3, Cz, C4), and posterior sites (P3, Pz, P4). 
nonprominent than in the prominent condition, possibly because memory retrieval as a function of the number of links in semantic memory that can be made was larger in this condition with the conjunctive nounphrase. Importantly, none of the reported effects can be attributed to the position of the repeated names in the sentence.

\section{DISCUSSION}

The results of this study support the view that discourse prominence impairs subsequent coreference with a repeated name. The N400 was larger in amplitude to a repeated name when its referent was prominent (singular noun phrase) than when it was not (conjunctive noun phrase). A larger N400 amplitude was found to repeated names when they were preceded by a prominent referent, even though the lag between the first and second presentation of the name in the sentence was smaller when the referent was prominent. In contrast, when words are repeated in lists, the amplitude of the $\mathrm{N} 400$ is smaller to words that are repeated with a smaller rather than with a greater lag (e.g., Nagy \& Rugg, 1989). Thus, the present study showed a reverse-lag effect where the amplitude of the N400 increased with proximity rather than with distance.

Importantly, this effect could not be attributed to differential expectation of the names in the prominent and nonprominent conditions (as determined by pretesting described in the Methods section), nor to the difference in sentential position. The reverse-lag effect is a new finding that shows that processing of coreferential relations invokes higher level processes that do not build in a straightforward way on basic lexical processing mechanisms. This result opens the possibility for further exploration of the nature of proximity and prominence in discourse processing.

Discourse prominence also had an effect on a positive shift that followed the N400. This positivity (LPC) was larger to repeated names in the nonprominent than in the prominent condition. This finding of a greater positivity for repeated names with a nonprominent referent is inconsistent with the predictions that were made with regard to the syntactic P600/SPS, because the repeated name in the nonprominent condition cannot be processed as a grammatical error, nor is the process of syntactic integration more difficult in this condition than in the prominent condition. However, this finding is consistent with Van Petten et al. (1991), who have suggested that an increase in the amplitude of the LPC to repeated names in congruent texts can be attributed to increased semantic knowledge about a referent when it is repeated. In our study, in the nonprominent condition the repeated name was embedded in a conjunctive noun phrase, and relative to the prominent condition, where the referent was a singular noun phrase, this may have increased the number of links in semantic memory that could be made and have therefore facilitated memory retrieval of the referent. Overall, the ERP results are consistent with the idea that coreference with a repeated name preceded by a prominent referent was not processed as a grammatical error, but instead show that coreferential integration with repeated names is more difficult with a prominent antecedent.

The results of this study also support the general view that constructing a mental representation of a discourse requires mechanisms specifically for keeping information appropriately integrated and differentiated. Coreference in a text provides one of the fundamental ways of integrating information from separate clauses into a coherent discourse representation. Language provides different options for conveying coreference in a text. Reduced expressions, such as pronouns, provide the natural vehicle for conveying coreference with a prominent referent because of the linguistic structure. In contrast, full expressions (such as repeated names) cannot easily convey coreference, especially when the referent is prominent in the discourse. This integration difficulty with repeated names can be explained in terms of what theoretical linguists call "disjoint reference" as described in Chomsky's (1981) binding theory. For example, in the sentence "John thinks that John is smart," the two occurrences of John seem like they must refer to different people, so the expressions are said to have disjoint reference. Recent analyses of linguistic and behavioral evidence (Gordon \& Hendrick, 1997, 1998) indicate that disjoint reference is a graded phenomenon that extends beyond the domain that theoretical linguists have traditionally conceived.

The difficulty in integration of repeated names in the prominent condition, shown by the N400 effect, can be seen as involving this tendency towards processing the repeated names as having disjoint reference. Of course, Chomsky's binding theory as a statement of grammatical rules does not give us a processing account of this phenomenon. Nor do all linguists accept the idea that disjoint reference with repeated names should be considered as part of grammar (Reinhart, 1983). For example, the difficulty in processing repeated names could be construed as a pragmatic phenomenon where the provision of too much information causes the reader to expend some processing resources in trying to determine why apparently unnecessary information was presented. Processing models of the phenomenon can be framed in terms of how a repeated name might increase the processing load on working memory or in terms of how a repeated name requires activation of an entity and then formulation of a coreferential link. The Gordon and Hendrick (1998) model takes this second view, arguing that the primary purpose of names is to introduce entities into a discourse model and that using repeated names for coreference requires a subsequent step of equating the new entity with an entity already represented in the discourse model. The ERP data presented 
do not distinguish between these detailed alternatives, but they do show that referential context has a strong effect on the ease of coreferential processing of repeated names.

Referential processing requires that different tokens of the same word either be integrated or kept distinct depending on the model of discourse, otherwise the meaning of the discourse is not adequately represented. Most of the word-processing paradigms used to study basic cognitive processes do not depend on distinguishing different tokens of a word, which in our judgment is the reason why the mechanisms invoked to explain performance in those paradigms does not extend in a straightforward way to the processing of coreference. Some recent research on memory has used paradigms where different tokens of a word must be kept distinct. This research has led to "ironic effects of repetition" where patterns of word repetition that facilitate performance in most tasks lead to worse performance (Jacoby, 1999). At a very broad level, such patterns are similar to the effects that we observed here on the neural processing of repeated words that are coreferentially related. It is an empirically open question whether the same mechanisms are used for keeping track of different tokens during language comprehension and during more general cognitive processing.

\section{METHODS}

\section{Subjects}

Participants were 20 right-handed native speakers of English (9 men, age 18 to 26, mean 21). All had normal or corrected-to-normal vision, and were recruited from the university population. All participants were unmedicated and neurologically unimpaired. None of the subjects that participated in the ERP experiment had taken part in the pretests (see below). Participants gave informed consent before the experiment and were compensated with payment or course credit.

\section{Materials}

A total of 160 experimental sentences were constructed. Each sentence could be assigned to any of the four conditions (prominent/pronoun, prominent/name, nonprominent/pronoun, nonprominent/name) by manipulating the noun phrase (single or conjunction) and coreferential expression (pronoun or repeated name) used. In the nonprominent condition where a conjunction of two names was used, one name was stereotypically female and one was stereotypically male. Examples of the stimuli are presented in Appendix A (a full set of materials can be obtained from the authors). The sentences contained two clauses that were connected with a temporal or causal conjunction (e.g., "when," "after," "so that"). Average length of the sentences was 12.4 words (range 8-18 words). The critical names were selected from a list of names that was used for a previous study (Gordon et al., 1999). Three native English speakers judged the names for gender bias. Only names with a clear gender bias were selected, so that pronoun assignment was unambiguous. The average length of the critical names was 4.9 characters (range 3-6). Each name was used only once within an experimental list.

The experimental sentences were mixed in with 80 filler sentences that had different structures than the experimental sentences to mitigate subject strategies. All of the 80 filler sentences contained names so that they would not be too distinct from the experimental sentences. Half of the fillers began with a word other than a name so that the subjects would not always expect the sentences to begin with a name. The other half of the fillers did begin with a single name or a conjunction of two names (like the experimental sentences), but for these fillers coreference was never established using a repeated name or a "he" or "she" pronoun.

\section{Pretest}

A pretest was conducted to test the cloze probability of the critical repeated names and the control pronouns in the prominent and nonprominent conditions. For this test, two questionnaires were prepared that included 50 representative experimental sentence fragments and 50 filler fragments. The fragments included the start of the sentences up to the critical name [e.g., John (and Mary) went to the store so that ]. Half of the filler fragments did not contain any names, and the other half did contain names but ended in such a way that the immediate continuation with a pronoun or repeated name would be awkward or impossible. Each list consisted of 25 experimental fragments that began with a conjunction noun phrase (nonprominent) and 25 that began with a single name (prominent). The sentence fragments were counterbalanced across the two lists such that fragments appearing in the "nonprominent" conjunction condition in list one would be in the "prominent" singular condition in list two, and vice versa. Twenty subjects who did not participate in the ERP experiment were asked to complete these sentence fragments with the first continuation that came to mind. The results are displayed in Table 1 and showed that the critical names had a very low cloze probability in both the prominent condition ( 1 of the 500 responses) and in nonprominent condition (3 of the 500 responses). Ninety percent of subjects gave no repeated name responses at all. Thus, the probability of a repeated name was equivalently low in the two conditions compared. In contrast, there was a high cloze probability for singular pronouns in the prominent condition ( 245 responses in $500)$ but not following the nonprominent condition ( 4 responses in 500). This pretest shows that differential expectation of the repeated name in the prominent and 
Table 1. Cloze Pretest for Sentences Beginning with a Singular or Conjunctive Noun Phrase

\begin{tabular}{|c|c|c|c|c|}
\hline & Singular Pronoun (He/She) & Plural Pronoun (They) & Repeated Name & Other \\
\hline Singular noun phrase & $49.0 \%$ & $1.6 \%$ & $0.2 \%$ & $47.4 \%$ \\
\hline Conjunction noun phrase & $0.8 \%$ & $43.8 \%$ & $0.6 \%$ & $54.8 \%$ \\
\hline
\end{tabular}

The percentage of continuations starting with a singular pronoun, plural pronoun, or repeated name are compared.

nonprominent conditions cannot be the basis of observed differences in ERPs.

\section{Randomization}

Four lists were created. The first list was created by pseudorandomly assigning 40 experimental sentences to each condition. Condition assignment in List 1 determined the assignment of experimental conditions for all four of the counterbalanced lists. For example, all of the sentences that used a conjunction NP and repeated name in List 1 were assigned conjunction NP and pronoun in List 2 , single NP and repeated name in List 3, and single NP and pronoun in List 4. Each list was then randomized separately.

\section{Procedure}

Each subject was tested individually in a dimly lit, electrically shielded sound-attenuating booth. They were seated in a comfortable chair approximately $100 \mathrm{~cm}$ from the computer screen, and were instructed to move as little as possible. They were asked to silently read the sentences and to answer a true or false question after each sentence by pressing one of two buttons on a button box with the index and middle fingers of their right or left hand. Response hand was counterbalanced across subjects.

Each trial began with a fixation cross that was presented for $1500 \mathrm{msec}$ in the center of the screen. The fixation cross alerted subjects to the beginning of a trial and showed them where to fixate during the upcoming sentence. Then a sentence was presented at a rate of 500 msec per word with an interstimulus interval (ISI) of $200 \mathrm{msec}$. The words were presented in the center of a computer screen as white letters against a dark background in lowercase with Tahoma, 14-point font. The first word of each sentence began with a capital letter, and the last word was presented together with a period. During the presentation of the experimental and filler sentences subjects were asked to keep their eyes fixated on the center of the screen and to refrain from blinking. The true or false question would appear $1500 \mathrm{msec}$ after the presentation of the sentence. While the true or false question was displayed, the subjects were permitted to move their eyes and blink. After subjects responded to the statement "press for next" a prompt appeared on the center of the screen. The subject started the next trial by pressing either button.

Each list was divided in eight blocks of 30 sentences, each block started with three warm-up filler sentences. After each block, subjects were given a short break.

\section{EEG Recording}

EEG was recorded from 30 tin electrodes fitted in an elastic cap (midline: $\mathrm{Fz}, \mathrm{FC}, \mathrm{Cz}, \mathrm{CP}, \mathrm{Pz}, \mathrm{Oz}$; lateral: Fp1/2, F3/4, F7/8, FC3/4, FT7/8, C3/4, T7/8, CP3/4, TP7/8, $\mathrm{P} 4 / 5, \mathrm{P} 7 / 8, \mathrm{O} 1 / 2)$ referenced to the left mastoid. Vertical and horizontal eye movements were monitored via suband supraorbital electrodes and left and right external canthus montages, respectively. Impedance was kept below $5 \mathrm{k} \Omega$. Prior to off-line averaging, all single-trial waveforms were automatically screened for amplifier blocking, muscle artifacts, horizontal eye movements, and blinks. This was done over an epoch of $1300 \mathrm{msec}$, starting $200 \mathrm{msec}$ before the onset of the critical words. For each subject, average ERPs were computed over artifact-free trials for the critical names, the critical pronouns in all four conditions (for all stimuli and for stimuli in a length constant analysis; see Results), and for the unrepeated second name in the conjunction condition. Off-line the waveforms were re-referenced to the algebraic average of both mastoids. The band pass was 0.01 to $30 \mathrm{~Hz}$ at a sampling rate of $250 \mathrm{~Hz}$.

\section{APPENDIX A}

Twenty examples of experimental stimuli in the four conditions used in this experiment.

1. Dirk (and Becca) pushed the desk into the corner because Dirk/he needed room for the filing cabinet.

2. Hannah (and Joey) purchased a new futon even though Hannah/she didn't really have room for it.

3. Myra (and Vic) decided to buy a new car since $\mathrm{Myra} / \mathrm{she}$ had wrecked the old one.

4. Irma (and Simon) explained the details while Irma/ she drew a diagram on the chalkboard.

5. Ronald (and Linda) remembered last year's party as Ronald/he wrapped the presents.

6. Freddy (and Monica) addressed the letters before Freddy/he stuck on the stamps.

7. Chase (and Mabel) looked for a ladder because Chase/he needed a box on the top shelf. 
8. Annie (and Derek) watched the clock strike midnight before Annie/she opened the champagne.

9. Lester (and Ellen) felt optimistic after Lester/he met the well-qualified job applicants.

10. Ruby (and Donny) accrued more library fines when Ruby/she returned the books late.

11. Harry (and Patsy) glanced at the subway schedule before Harry/he bought the tokens.

12. Jan (and Ben) picked up the sticks so that Jan/she could mow the lawn.

13. Eliott (and Muriel) arranged the new dining room table so that Eliott/he could see out the window.

14. Trevor (and Karen) strained to hear the speaker before Trevor/he fixed the sound system.

15. Amber (and Rod) sang scales until Amber/she was warmed up enough for the lesson.

16. Andrea (and Herb) searched for an outlet while Andrea/she unwound the extension cord.

17. Abbie (and Pete) booked a hotel room as soon as Abbie/she knew the wedding date.

18. Philip (and Emma) shredded the documents after Philip/he entered the secure data.

19. Bud (and Marge) read the instructions before $\mathrm{Bud} /$ he assembled the chair.

20. Margie (and Adam) entered the office as soon as Margie/she unlocked the door.

\section{Acknowledgments}

This research was supported by NSF grants SES-0074634 to TYS, BCS-0112231 to PCG, and NIMH grant R01 MH066271 to PCG and TYS. We thank Adriane Boyd, Joe Hopfinger, and Edith Kaan for helpful discussions.

Reprint requests should be sent to Tamara Y. Swaab, Department of Psychology, UC Davis, Davis, CA 95616, USA, or via e-mail: swaab@ucdavis.edu; or to Peter C. Gordon, Department of Psychology, University of North Carolina, Chapel Hill, NC 27599-3270, USA, or via e-mail: pcg@email.unc.edu.

\section{Notes}

1. In order to make sure that the effects of discourse prominence were independent of choice of baseline, we repeated all ANOVAs that were done for the 100-msec prestimulus baseline with a 100-msec poststimulus baseline, and we obtained essentially the same pattern of results: In the 250to 500 -msec time window, there was a main effect of discourse prominence, $F(1,19)=6.40, p<.025$, as well as a marginal prominence by electrode interaction, $F(29,551)=2.73$, $p=.052$; there was a significant prominence effect at the posterior electrodes, $F(1,19)=9.62, p<.01$. In the 500 - to 700 -msec time window there was a main effect of discourse prominence, $F(1,19)=4.97, p=.038$, a prominence by electrode site interaction, $F(29,551)=2.48, p=.038$, and also a significant effect of prominence when analysis was limited to posterior sites, $F(1,19)=8.47, p=.009$.

2 . In order to make sure that these effects were again independent of choice of baseline (see also note 1), we repeated all the ANOVAs for this repeated and nonrepeated name analysis with a poststimulus baseline of $100 \mathrm{msec}$. Overall these analyses pointed in the same direction as those with the prestimulus baseline: In the $250-$ to $500-\mathrm{msec}$ time window there was no significant effect of repetition over all electrodes, $F(1,19)=1.92, p=.18$, a significant repetition by electrode interaction, $F(29,551)=3.58, p<.006$, and no main effect of repetition at the posterior electrodes, $F(1,19)=$ $3.55, p=.075$. In the 500 - to $700-\mathrm{msec}$ time window there was no main effect of repetition over all electrodes $(F<1)$, a significant interaction of repetition by electrode, $F(29,551)=$ $1.66, p<.025$, and no main effect of repetition at posterior electrodes $(F<1)$.

\section{REFERENCES}

Almor, A. (1999). Noun-phrase anaphora and focus: The informational load hypothesis. Psychological Review, 106, 748-765.

Bousfield, W. A. (1953). The occurrence of clustering in the recall of randomly arranged associates. Journal of General Psychology, 49, 229-240.

Brown, C. M., \& Hagoort, P. (1993). The processing nature of the N400: Evidence from masked priming. Journal of Cognitive Neuroscience, 5, 34-44.

Chang, F. R. (1980). Active memory processes in visual sentence comprehension: Clause effects and pronominal reference. Memory and Cognition, 8, 58-64.

Chomsky, N. (1981). Lectures on government and binding. Dordrecht, the Netherlands: Foris.

Chwilla, D. J., Brown, C. M., \& Hagoort, P. (1995). The N400 as a function of the level of processing. Psychophysiology. 32, 274-85

Cloitre, M., \& Bever, T. G. (1988). Linguistic anaphors, levels of representation, and discourse. Language and Cognitive Processes, 3, 293-322.

Collins, A. M., \& Loftus, E. F. (1975). A spreading activation theory of semantic processing. Psychological Review, 82, 407-428.

Federmeier, K. D., \& Kutas, M. (1999). A rose by any other name: Long-term memory structure and sentence processing. Journal of Memory and Language, 41, 469-495.

Garrod, S., Freudenthal, D., \& Boyle, E. A. (1994). The role of different types of anaphor in the on-line resolution of sentences in a discourse. Journal of Memory and Language, 33, 39-68.

Gernsbacher, M. A. (1989). Mechanisms that improve referential access. Cognition, 32, 99-156.

Gernsbacher, M. A. (1990). Language comprehension as structure building. Hillsdale, NJ: Erlbaum.

Gernsbacher, M. A. (1996). The structure-building framework: What it is, what it might also be, and why. In B. K. Britton \& A. C. Graesser (Eds.), Models of understanding text (pp. 289-312). Mahwah, NJ: Erlbaum.

Gillund, G., \& Shiffrin, R. M. (1984). A retrieval model for both recognition and recall. Psychological Review, 19, 1-65.

Gordon, P. C., Grosz, B. J., \& Gilliom, L. A. (1993). Pronouns, names, and the centering of attention in discourse. Cognitive Science, 17, 311-347.

Gordon, P. C., \& Hendrick, R. (1997). Intuitive knowledge of linguistic coreference. Cognition, 62, 325-370.

Gordon, P. C., \& Hendrick, R. (1998). The representation and processing of coreference in discourse. Cognitive Science, 22, 389-424.

Gordon, P. C., Hendrick, R., \& Foster, K. (2000). Language comprehension and probe-list memory. Journal of Experimental Psychology: Learning, Memory, and Cognition, 26, 766-775.

Gordon, P. C., Hendrick, R., Ledoux, K., \& Yang, C. L. (1999). Processing of reference and the structure of language: 
An analysis of complex noun phrases. Language and Cognitive Processes, 14, 353-379.

Greene, S. B., McKoon, G., \& Ratcliff, R. (1992). Pronoun resolution and discourse models. Journal of Experimental Psychology: Learning, Memory, and Cognition, 18, 266-283.

Greenhouse, S. W., \& Geisser, S. (1959). On methods in the analysis of profile data. Psychometrika, 24, 95-112.

Hagoort, P., Brown, C., \& Groothusen, J. (1993). The syntactic positive shift (SPS) as an ERP-measure of syntactic processing. Language and Cognitive Processes, 8, 439-484.

Holcomb, P. J. (1993). Semantic priming and stimulus degradation: Implications for the role of the N400 in language processing. Psychophysiology, 30, 47-61.

Hudson, S. B., Tanenhaus, M. K., Dell, G. S. (1986). The effect of the discourse center on the local coherence of a discourse. In Proceedings of the 8th Annual Conference of the Cognitive Science Society (pp. 96-101). Hillsdale, $\mathrm{NJ}$ : Erlbaum.

Jacoby, L. L. (1999). Ironic effects of repetition: Measuring age-related difference in memory. Journal of Experimental Psychology: Learning, Memory, and Cognition, 25, 3-22.

Kaan, E., \& Swaab, T. Y. (2003). Repair, revision and complexity in syntactic analysis: An electrophysiological differentiation. Journal of Cognitive Neuroscience, 15, 98-110.

Kennison, S. M., \& Gordon, P. C. (1997). Comprehending referential expressions during reading: Evidence from eye tracking. Discourse Processes, 24, 229-252.

Kintsch, W., \& van Dijk, T. A. (1978). Toward a model of text comprehension and production. Psychological Review, 85, 363-394.

Kounios, J., \& Holcomb, P. J. (1992). Structure and process in semantic memory: Evidence from event-related brain potentials and reaction times. Journal of Experimental Psychology: General, 121, 459-479.

Kutas, M. (1993). In the company of other words: Electrophysiological evidence for single-word and sentence context effects. Language and Cognitive Processes, 8, 533-572.

Kutas, M., \& Hillyard, S. A. (1980). Reading senseless sentences: Brain potentials reflect semantic incongruity. Science, 207, 203-205.

Kutas, M., \& Hillyard, S. A. (1989). An electrophysiological probe of incidental semantic association. Journal of Cognitive Neuroscience, 1, 38-49.

MacDonald, M. C., \& MacWhinney, B. (1990). Measuring inhibition and facilitation from pronouns. Journal of Memory and Language, 29, 469-492.

Meyer, D. E., \& Schvaneveldt, R. W. (1971). Facilitation in recognizing pairs of words: Evidence of a dependency in retrieval operations. Journal of Experimental Psychology, 90, 227-234.

Morton, J. (1969). Interaction of information in word recognition. Psychological Review, 76, 165-178.

Nagy, M. E., \& Rugg, M. D. (1989). Modulation of event-related potentials by word repetition: The effects of inter-item lag. Psychophysiology, 26, 431-436.

Osterhout, L., Bersick, M., \& McKinnon, R. (1997). Brain potentials elicited by words: Word length and frequency predict the latency of an early negativity. Biological Psychology, 46, 143-168.
Osterhout, L., \& Holcomb, P. J. (1992). Event-related brain potentials elicited by syntactic anomaly. Journal of Memory and Language, 31, 785-806.

Osterhout, L., \& Mobley, L. A. (1995). Event-related brain potentials elicited by failure to agree. Journal of Memory and Language, 34, 739-773.

Paller, K. A., Kutas, M., \& McIsaac, H. K. (1995). Monitoring conscious recollection via the electrical activity of the brain. Psychological Science, 6(2), 107-111.

Reinhart, T. (1983). Anaphora and semantic interpretation. London: Croom Helm.

Rips, L., Shoben, E. J., \& Smith, E. E. (1973). Semantic distance and the verification of semantic relations. Journal of Verbal Learning and Verbal Behavior, 12, 1-20.

Rugg, M. D. (1985). The effects of semantic priming and word repetition on event-related potentials. Psychophysiology, 22, 642-647.

Rugg, M. D. (1990). Event-related brain potentials dissociate repetition effects of high- and low-frequency words. Memory and Cognition, 18, 367-379.

Rugg, M. D., \& Doyle, M. C. (1992). Event-related potentials and recognition memory for low- and high-frequency words. Journal of Cognitive Neuroscience, 4, 69-79.

Rugg, M. D., Furda, J., \& Lorist, M. (1988). The effects of task on the modulation of event-related potentials by word repetition. Psychophysiology, 25, 55-63.

Sanford, A. J., \& Garrod, S. C. (1981). Understanding written language: Explorations of comprehension beyond the sentence. New York: Wiley.

Scarborough, D. L., Cortese, C., \& Scarborough, H. S. (1977). Frequency and repetition effects in lexical memory.

Journal of Experimental Psychology: Human Perception and Performance, 3, 1-17.

Seidenberg, M. S., Tanenhaus, M. K., Leiman, J. M., \& Bienkowski, M. (1982). Automatic access of the meanings of ambiguous words in context: Some limitations of knowledge-based processing. Cognitive Psychology, 14, 489-537.

Swinney, D. A. (1979). Lexical access during sentence comprehension: (Re)consideration of context effects. Journal of Verbal Learning and Verbal Behavior, 18, 645-659.

van Berkum, J. J. A., Hagoort, P., \& Brown, C. M. (1999). Semantic integration in sentences and discourse: Evidence from N400. Journal of Cognitive Neuroscience, 11(6), 657-671.

Van Petten, C. (1993). A comparison of lexical and sentencelevel context effects in event-related potentials. Language and Cognitive Processes, 8, 485-531.

Van Petten, C., \& Kutas, M. (1991). Influences of semantic and syntactic context on open- and closed-class words. Memory and Cognition, 19, 95-112.

Van Petten, C., Kutas, M., Kluender, R., Mitchiner, M., McIsaac, H. (1991). Fractionating the word repetition effect with event-related potentials. Journal of Cognitive Neuroscience, 3, 131-150.

Wilding, E. L., Doyle, M. C., \& Rugg, M. D. (1995). Recognition memory with and without retrieval of context: An event-related potential study. Neuropsychologia, 33, 743-767. 